\title{
An image decomposition model using the total variation and the infinity Laplacian
}

\author{
Christopher Elion ${ }^{a}$ and Luminita A. Vese ${ }^{a}$ \\ ${ }^{a}$ Department of Mathematics, University of California Los Angeles, \\ 405 Hilgard Avenue, Los Angeles, CA 90095-1555, U.S.A.
}

\begin{abstract}
This paper is devoted to a recent topic in image analysis: the decomposition of an image into a cartoon or geometric part, and an oscillatory or texture part. Here, we propose a practical solution to the $(\mathrm{BV}, \mathrm{G})$ model proposed by Y. Meyer ${ }^{1}$. We impose that the cartoon is a function of bounded variation, while the texture is represented as the Laplacian of some function whose gradient belongs to $L^{\infty}$. The problem thus becomes related with the absolutely minimizing Lipschitz extensions and the infinity Laplacian. Experimental results for image denoising and cartoon + texture separation, together with details of the algorithm, are also presented.
\end{abstract}

Keywords: functional minimization, partial differential equation, image denoising, image decomposition, texture modeling, sup norm.

\section{INTRODUCTION}

We limit the presentation to two dimensions and gray-scale images, but any number of dimensions and vectorvalued data can be considered. Let $\Omega$ be an open, bounded, and connected domain in $R^{2}$ with Lipschitzcontinuous boundary, and $f: \Omega \rightarrow R$ a given image-function, the observation. Sometimes we will assume that $f: R^{2} \rightarrow R$. Often the image $f$ has been corrupted by noise and/or blur. Important problems in image analysis are, for instance, the inverse reconstruction problem, the extraction of information and of main features, or the separation of different features. Mathematically this is often related with $K$-functionals or $J$-functionals in the theory of interpolation between spaces, and amounts to find for $f \in X_{1}+X_{2}$ optimal pairs $(u, v) \in X_{1} \times X_{2}$ by solving one of the equivalent minimizations

$$
\inf _{f=u+v, u \in X_{1}, v \in X_{2}}\left\{\|u\|_{X_{1}}+\lambda\|v\|_{X_{2}}\right\}=\inf _{u \in X_{1}}\left\{\|u\|_{X_{1}}+\lambda\|f-u\|_{X_{2}}\right\},
$$

where $X_{1}$ and $X_{2}$ are spaces of functions or of distributions. More generally, we can formulate decompositions $f=u+v$ via energy minimization

$$
\inf _{(u, v) \in X_{1} \times X_{2}}\left\{\mathcal{K}(u, v)=F_{1}(u)+\lambda F_{2}(v): f=u+v\right\},
$$

where $F_{1}, F_{2} \geq 0$ are functionals such that $X_{1}=\left\{u: F_{1}(u)<\infty\right\}, X_{2}=\left\{v: F_{2}(v)<\infty\right\}$ (for example $\left.F_{i}(\cdot)=\|\cdot\|_{X_{i}}\right)$, and $f \in X_{1}+X_{2}$. The constant $\lambda>0$ is a tuning parameter. For instance, in image denoising, $f$ is the observed noisy version of the true unknown image $u$, while $v$ represents additive noise of zero mean. In this case $X_{1} \subset X_{2}, f \in X_{2}$ and $X_{1}$ is a space of functions "smoother" or less oscillating than those in $X_{2}$. However, sharp edges or boundaries have to be represented in $u$. Another related problem is the separation of the geometric (cartoon) component $u$ of $f$ from the oscillatory component $v$, representing texture or noise of zero mean. In other cases, $u$ can be seen as a structure component of $f$, while $v$ is clutter ${ }^{2}$ (where $f$ is the image of a building occluded by trees, $u$ contains only the building from $f$ and $v$ contains only the trees). A good model for $\mathcal{K}$ is given by a choice of $X_{1}$ and $X_{2}$ so that with the above given properties of $u$ and $v$, the values $F_{1}(u)$ and $F_{2}(v)$ are small, and $F_{1}(v)>F_{1}(u), F_{2}(u)>F_{2}(v)$.

Further author information: (Send correspondence to L.A.V.)

C.E.: E-mail: elion@math.ucla.edu

L.A.V.: E-mail: lvese@math.ucla.edu

Computational Imaging V, edited by Charles A. Bouman, Eric L. Miller, llya Pollak

Proc. of SPIE-IS\&T Electronic Imaging, SPIE Vol. 6498, 64980W, @ 2007 SPIE-IS\&T · 0277-786X/07/\$18 
Variational models in image restoration by regularization can be seen as decomposition models. Indeed, consider the canonical model: given $f \in L^{2}(\Omega)$, solve

$$
\inf _{u}\left\{F(u)=\int_{\Omega} \phi(|D u|) d x+\lambda\|f-u\|_{L^{2}(\Omega)}^{2}\right\}
$$

where $\phi:[0, \infty) \rightarrow[0, \infty)$ is a continuous potential, increasing, with at most linear growth at infinity (to allow discontinuities along curves); examples are $\phi(t)=\frac{t^{2}}{1+t^{2}}, \frac{|t|}{1+|t|}, \log \left(1+t^{2}\right)$ in the non-convex case, $|t|$, $\sqrt{1+t^{2}}, \log \cosh \left(1+t^{2}\right)$ in the convex case. The unknown minimizer $u$ has to be recovered from $f$, assuming the degradation model $f=u+$ noise. For more details on this class of models we refer the reader to ${ }^{3},{ }^{4},{ }^{5},{ }^{6},{ }^{7}$ $,{ }^{8},{ }^{9}$. Model (1) can be formulated as an image decomposition model,

$$
\inf _{(u, v), f=u+v} F(u, v)=\int_{\Omega} \phi(|D u|) d x+\lambda\|v\|_{L^{2}(\Omega)}^{2} .
$$

The model should produce a cartoon or piecewise-smooth component $u$ (geometric component) and an additive oscillatory component of zero mean $v \in L^{2}(\Omega)$. In the convex case, the above functional is well defined and has a unique minimizer on the space of functions of bounded variation $B V(\Omega)^{10},{ }^{9},{ }^{6},,^{7},{ }^{11}$. The case $\phi(t)=|t|$ corresponds to the total variation model (L. Rudin, S. Osher, E. Fatemi ${ }^{12},{ }^{5},{ }^{13}$ ), often called "ROF" model,

$$
\inf _{(u, v) \in L^{2}(\Omega) \times B V(\Omega)}\left\{\int_{\Omega}|D u|+\lambda\|v\|_{L^{2}(\Omega)}^{2}, f=u+v\right\}
$$

and will be of particular interest.

When $v$ is additive Gaussian noise of zero mean, of uniform variance, the space $v \in L^{2}(\Omega)$ is the natural choice. Also, having $u \in B V(\Omega)$ insures that $u$ will be made of homogeneous regions with sharp boundaries. However, there are some limitations of model (2). For instance, it can be shown ${ }^{14},{ }^{1},{ }^{11}$ that, even if the initial data $f$ is the characteristic function of a smooth convex domain with finite perimeter, without noise, the solution $u$ of the model (2) is not exactly $f$ (therefore, the residual $v=f-u$ is not zero): the ROF model does not always correctly separate oscillations from $B V$ components. On the other hand, Mumford and Gidas ${ }^{15}$ mention that natural images, containing texture, noise, clutter, and other oscillatory details, cannot be modeled by functions, but rather by distributions, or generalized functions. Also, in Gousseau, Morel ${ }^{16}$, Alvarez, Gousseau, Morel ${ }^{17}$ it is shown that natural images (with textured details of different scales) are not well modeled by functions of bounded variation. On the other hand, the ROF model and all models in the class (1) only keep $u$, a piecewisesmooth function, and treat $v$ as noise or residual. Similarly, in the non-convex Mumford and Shah model ${ }^{18},{ }^{19}$ for computing optimal piecewise-smooth approximations $u$ of $f$, only the cartoon component $u$ is kept.

Thus we have to relax the conditions on $u$ or on $v$ in the model (2), and to model images by functions in larger spaces. One way to modify (2) is to keep the $L^{2}$ norm and to work with non-convex potentials $\phi$. However, in this case, the problem is theoretically open and may not have a solution. Thus we prefer to keep the $B V$ regularization, and we follow Meyer's idea ${ }^{1}$, to work with weaker norms than the $L^{2}$ norm for $v$.

We propose here a new method, which allows to model textures using partial differential equations and oscillating patterns. A new task in image analysis is introduced, called image decomposition into cartoon + texture, following a proposal of Y. Meyer ${ }^{1}$. The main idea is to model the residual $v=f-u$ as a generalized function, in a space of distributions more appropriate for oscillations. In order to better extract both the $u$ component in $B V$ and the $v$ component as an oscillating function (texture in natural images or noise) from $f$, Meyer ${ }^{1}$ proposes in $R^{2}$, among several choices, the following space for $v$, larger than $L^{2}\left(R^{2}\right)$, denoted by $G$,

Definition 1.1. Let $G\left(R^{2}\right)$ be the Banach space of distributions $v=\operatorname{div} \vec{g}, g_{1}, g_{2} \in L^{\infty}\left(R^{2}\right)$, endowed with the norm

$$
\|v\|_{G\left(R^{2}\right)}=\inf _{\vec{g}=\left(g_{1}, g_{2}\right) \in\left(L^{\infty}\left(R^{2}\right)\right)^{2}, v=\operatorname{div} \vec{g}}\|\mid \vec{g}\|_{L^{\infty}\left(R^{2}\right)} .
$$


Y. Meyer proposes in the same lecture notes ${ }^{1}$ the following image decomposition model into "cartoon and texture":

$$
\inf _{(u, v) \in(B V \times G)}\left\{E(u)=\int|D u|+\lambda\|v\|_{G}, f=u+v\right\} .
$$

As explained in ${ }^{1}$, the space $G$ allows for oscillating functions $v$, and the oscillations are well measured by the norm $\|v\|_{G}$. Also, we can say that the space $G$ is, roughly speaking, the dual of $B V$ (this is not exactly true, but each one of the spaces $B V$ and $G$ is the dual of a subset of the other one). The space $G$ can be isometrically and isomorphically identified with the dual of $\left\{u \in B \dot{B}\left(R^{2}\right): D u \in L^{1}\left(R^{2}\right)\right\}$ (working with homogeneous versions).

However, it is not possible to directly solve and use the model (3) in practice, because we cannot express the Euler-Lagrange equation associated with the minimization problem, due to the definition of the G-norm. Motivated by the following approximation to the $L^{\infty}(\Omega)$ norm of $|\vec{g}|,\left\|\sqrt{g_{1}^{2}+g_{2}^{2}}\right\|_{L^{\infty}(\Omega)}=\lim _{p \rightarrow \infty}\left\|\sqrt{g_{1}^{2}+g_{2}^{2}}\right\|_{L^{p}(\Omega)}$, a first direction in approximating (3) has been proposed $\mathrm{in}^{20},{ }^{21}$ by the following minimization with a Lagrange multiplier $\mu$ for the constraint,

$$
\inf _{u, g_{1}, g_{2}}\left\{G_{p}\left(u, g_{1}, g_{2}\right)=\int_{\Omega}|D u|+\mu \int_{\Omega}|f-u-\operatorname{div} \vec{g}|^{2} d x+\lambda\||\vec{g}|\|_{L^{p}(\Omega)},\right.
$$

where $\lambda, \mu>0$ are tuning parameters, and $1<p<\infty$. The first term insures that $u \in B V(\Omega)$, the second term insures that $f \approx u+\operatorname{div} \vec{g}$, while the third term is a penalty on the norm of $v=\operatorname{div} \vec{g}$ : if $\mu \rightarrow \infty$ and $p \rightarrow \infty$, this model is an approximation of the model (3) originally proposed by Meyer (by this model, $v=\operatorname{div} \vec{g} \in L^{2}(\Omega) \cap G_{p}(\Omega)$, with $\left.\|v\|_{G_{p}(\Omega)}=\inf _{v=\operatorname{div} \vec{g}, g_{i} \in L^{p}(\Omega)}\|\vec{g}\|_{L^{p}(\Omega)}\right)$. Formally minimizing (4) with respect to the unknowns, leads to a coupled system of three non-linear Euler-Lagrange equations in $u, g_{1}, g_{2}$. In the case $p=2$, the norm $\|v\|_{G_{p}(\Omega)}$ coincides with the norm in $\dot{H}^{-1}(\Omega)$, dual to the homogeneous $\dot{H}^{1}(\Omega)$ space endowed with $\|u\|_{\dot{H}^{1}(\Omega)}=\int_{\Omega}|D u|^{2} d x^{22}$. This case $p=2$ has been modified $\operatorname{in}^{23}$ where $v=\operatorname{div} \vec{g}=\operatorname{div}(D P)$, with $P$ a scalar function and producing an exact $\left(B V, \dot{H}^{-1}\right)$ decomposition model $f=u+\triangle P$, by

$$
\inf _{u \in B V(\Omega)} \int_{\Omega}|D u|+\lambda \int_{\Omega}\left|D \triangle^{-1}(f-u)\right|^{2} d x
$$

(this is also related with another $(B V, E)$ model proposed by $\mathrm{Y}$. Meyer ${ }^{1}$, where $v=\triangle P, P \in \dot{B}_{\infty, \infty}^{1}$ ).

In the work of Aujol et al. ${ }^{24},{ }^{25},{ }^{26}$, another approximation has been proposed to the $(B V, G)$ model: again $v \in L^{2}(\Omega) \cap G(\Omega)$, and minimizing

$$
\inf _{(u, v) \in B V(\Omega) \times G_{\mu}(\Omega)} \int_{\Omega}|D u|+\frac{1}{2 \lambda}\|f-u-v\|_{L^{2}(\Omega)}^{2},
$$

where $G_{\mu}(\Omega)=\left\{v \in G(\Omega):\|v\|_{G(\Omega)} \leq \mu\right\}$. Duality results are used to solve this model in practice.

Note that the expression $v=\operatorname{div} \vec{g}$ introduces numerically some anisotropy (unlike $v=\triangle P$ ), as observed in ${ }^{27}$ where another approximation to Meyer's $(B V, G)$ model was proposed:

$$
\inf _{(u, \vec{g}) \in B V(\Omega) \times L^{\infty}\left(\Omega, R^{2}\right)}\left\{\int_{\Omega}|D u|+\mu \int_{\Omega}|f-u-\operatorname{div}(\vec{g})|^{2} d x+\lambda \int_{\Omega} \sqrt{g_{1}(x)^{2}+g_{2}(x)^{2}} \delta\left(x-x_{0}\right) d x\right\} .
$$

Here $\delta$ is the Dirac function (an impulse function) in two dimensions concentrated at the origin, and

$$
\sqrt{g_{1}\left(x_{0}\right)^{2}+g_{2}\left(x_{0}\right)^{2}}=\|\mid \vec{g}\|_{L^{\infty}(\Omega)} .
$$

A numerical computation has been proposed in ${ }^{27}$ by alternating minimization (at each step $n \geq 0$, if $u^{n}, g_{i}^{n}$ are known, let $x_{0}=\operatorname{argmax}_{x \in \Omega}|\vec{g}(x)|$; then $x_{0}$ is kept fixed and the energy to be minimized in $u=u^{n+1}, g_{i}=g_{i}^{n+1}$ is now of integral form and associated Euler-Lagrange equations can be expressed; update $x_{0}$ and repeat).

More recently, other solutions have been proposed to solve $(B V, G)$ decomposition models, including ${ }^{28}$ using second order cone programming, and ${ }^{29}$ using duality results. In this paper we would like to propose another "isotropic" decomposition $f \approx u+\triangle P=\operatorname{div}(D P)$, with $\vec{g}=D P \in L^{\infty}(\Omega)^{2}$. To minimize the sup norm of $|D P|$, we make use of the infinity Laplacian ${ }^{30}, 31,32$. 


\section{DESCRIPTION OF THE PROPOSED MODEL}

Given $f \in L^{2}(\Omega)$, the proposed new model is

$$
\inf _{u \in B V(\Omega), D P \in L^{\infty}(\Omega)^{2}, \triangle P \in L^{2}(\Omega)} F(u, P)=\int_{\Omega}|D u|+\mu \int_{\Omega}|f-(u+\triangle P)|^{2} d x+\lambda\|D P\|_{L^{\infty}(\Omega)} .
$$

In the limit, as $\mu \rightarrow \infty$, we have $f=u+\triangle P$, with $u \in B V(\Omega)$ and $v \in\{v=\triangle P=\operatorname{div}(D P), D P \in$ $\left.L^{\infty}(\Omega)^{2}\right\}$, with norm $\|v\|=\inf _{\left\{P, v=\triangle P=\operatorname{div}(D P), D P \in L^{\infty}(\Omega)^{2}\right\}}\|\mid D P\|_{L^{\infty}(\Omega)}$ (based on the assumption of the Hodge decomposition of any $\vec{g} \in L^{\infty}(\Omega)$ into $\vec{g}=D P+\vec{Q}$, where $P$ is a scalar function and $\vec{Q}$ is a divergencefree vector field, thus $\operatorname{div} \vec{g}=\triangle P$ ).

To minimize the above energy, we make here use of the so-called absolutely minimizing Lipschitz extensions AMLE ( ${ }^{30},{ }^{31}$, introduced in image analysis in $\left.{ }^{32}\right)$, the difficulty being due to the last term. We first recall the AMLE notion: given a bounded, open and connected domain $\Omega$ with sufficiently smooth boundary, and boundary data $\phi$ on $\partial \Omega$, solve

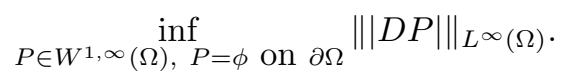

A minimizer $P$ is called an absolutely minimizing Lipschitz extension of $\phi$ into $\Omega$. It is shown that

$$
P_{x_{1}}^{2} P_{x_{1} x_{1}}+2 P_{x_{1}} P_{x_{2}} P_{x_{1} x_{2}}+P_{x_{2}}^{2} P_{x_{2} x_{2}}=0 \text { in } \Omega
$$

is the Euler-Lagrange equation for the minimal Lipschitz extension problem, in the sense of viscosity solutions or smooth solutions $P$ (using the notation $P_{x_{i}}=\frac{\partial P}{\partial x_{i}}$ ). $\operatorname{In}^{32}$ time-dependent versions of this problem have been used for image interpolation:

$$
\begin{aligned}
\frac{\partial P}{\partial t} & =P_{x_{1}}^{2} P_{x_{1} x_{1}}+2 P_{x_{1}} P_{x_{2}} P_{x_{1} x_{2}}+P_{x_{2}}^{2} P_{x_{2} x_{2}} \text { in }(0, \infty) \times \Omega, \\
P(0, x) & =P_{0}(x) \text { in } \Omega, \\
P(t, x) & =\phi(x) \text { for }(t, x) \in(0, \infty) \times \partial \Omega,
\end{aligned}
$$

together with its "normalized version"

$$
\begin{aligned}
\frac{\partial P}{\partial t} & =\frac{P_{x_{1}}^{2}}{|D P|^{2}} P_{x_{1}, x_{1}}+2 \frac{P_{x_{1}} P_{x_{2}}}{|D P|^{2}} P_{x_{1}, x_{2}}+\frac{P_{x_{2}}^{2}}{|D P|^{2}} P_{x_{2}, x_{2}} \text { in }(0, \infty) \times \Omega, \\
P(0, x) & =P_{0}(x) \text { in } \Omega, \\
P(t, x) & =\phi(x) \text { for }(t, x) \in(0, \infty) \times \partial \Omega .
\end{aligned}
$$

The operator $D^{2} P\left(\frac{D P}{|D P|}, \frac{D P}{|D P|}\right)=\frac{P_{x_{1}}^{2}}{|D P|^{2}} P_{x_{1} x_{1}}+2 \frac{P_{x_{1}} P_{x_{2}}}{|D P|^{2}} P_{x_{1} x_{2}}+\frac{P_{x_{2}}^{2}}{|D P|^{2}} P_{x_{2} x_{2}}=\triangle_{\infty} P$ represents the second order derivative of $P$ in the normalized gradient direction $\frac{D P}{|D P|}$.

(7) can be seen as the limiting case $p \rightarrow \infty$ of the inhomogeneous Dirichlet problem with $1<p<\infty$

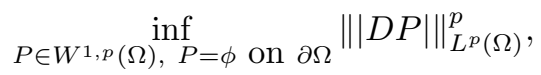

whose Euler-Lagrange equation is $\triangle_{p} u=0$ in $\Omega$. This limiting process $p \rightarrow \infty$ is made rigorous in ${ }^{30},{ }^{31}$.

In our case, we associate homogeneous Neumann boundary conditions to $P$ on $\partial \Omega$, to insure that $\int_{\Omega} \triangle P d x=$ 0 . Therefore, by the above results, we obtain the following Euler-Lagrange equations, in a time-dependent gradient descent method, to minimize (6):

$$
\begin{aligned}
\frac{\partial u}{\partial t} & =2 \mu(f-u-\triangle P)+\operatorname{div}\left(\frac{D u}{|D u|}\right), \\
\frac{\partial P}{\partial t} & =2 \mu \triangle(f-u-\triangle P)+\lambda\left(\frac{P_{x_{1}}^{2}}{|D P|^{2}} P_{x_{1} x_{1}}+2 \frac{P_{x_{1}} P_{x_{2}}}{|D P|^{2}} P_{x_{1} x_{2}}+\frac{P_{x_{2}}^{2}}{|D P|^{2}} P_{x_{2} x_{2}}\right), \\
P(0, x) & =P_{0}(x), \text { in }(0, \infty) \times \Omega, \\
u(0, x) & =f(x), \text { in }(0, \infty) \times \Omega,
\end{aligned}
$$



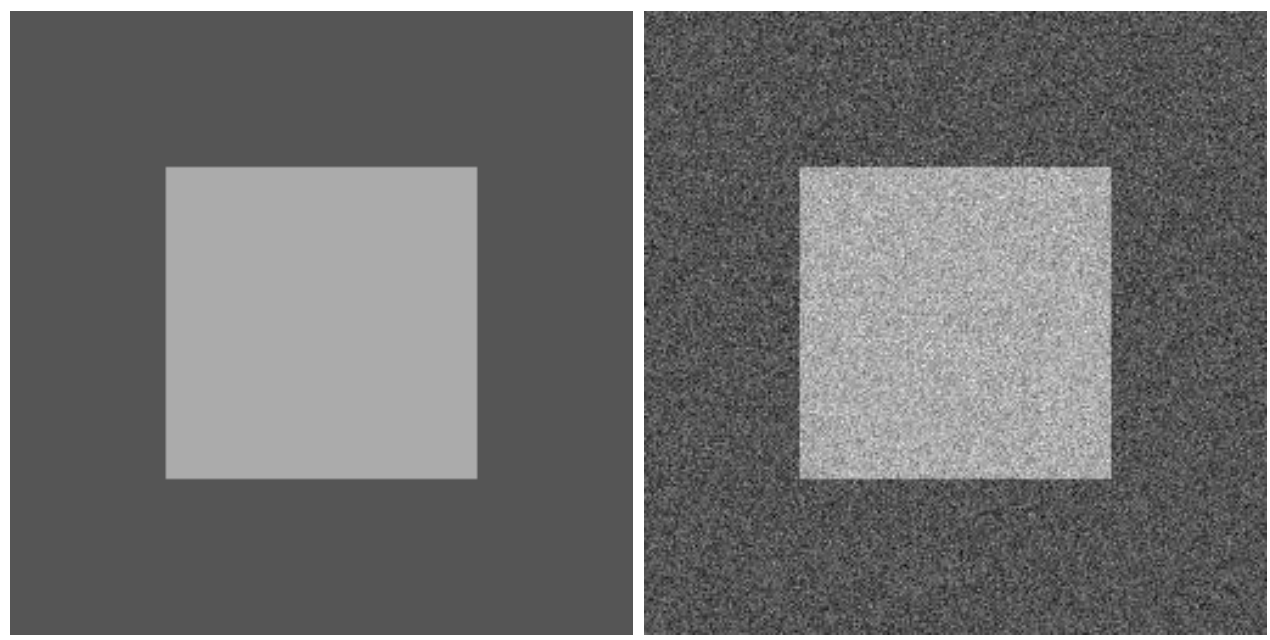

Figure 1. Synthetic original image and its noisy version $f$.

and with associated boundary conditions for $u, P$ and $(f-u-\triangle P)$ on $\partial \Omega$. In practice, this coupled system of partial differential equations is solved by finite differences to steady state. The numerical time-step $\Delta t$ is chosen such that a discrete version of the energy (6) is decreasing at each iteration. The initial $P_{0}$ is chosen to be identically zero, or an arbitrary (almost random) periodic pattern. Note that the proposed method has only two unknowns $u, P$, instead of three unknowns $u, g_{1}, g_{2}{ }^{20},{ }^{27}$ and insures that $v=\triangle P \in L^{2}(\Omega) \cap G(\Omega)$.

\section{EXPERIMENTAL RESULTS}

We present in this section several experimental results for image denoising and cartoon and texture decomposition using the proposed method (6), (9). Comparisons with the ROF model ${ }^{5}$ are also given in the denoising case, showing that the new model gives improved results, as expected. We also show plots of the discrete energy versus iterations. In practice, the residual $f-u-\triangle P$ is small, but not exactly zero. Thus we incorporate this small residual in the component $v$, therefore for the oscillatory component we plot $f-u$ instead of only $\triangle P$ (this still gives us an $f-u \in G(\Omega)$, because $f-u=\triangle P-\frac{1}{2 \mu} \operatorname{div}\left(\frac{D u}{|D u|}\right) \in G(\Omega)$ ).

In Figures 1 and 2 we show two original images and their noisy versions. In Figure 3 we show a denoised result $u$ of the synthetic square image using the proposed method, and the oscillatory component $f-u$, together with the obtained root mean square error (RMSE) and the signal-to-noise ratio (SNR); we also show here the plot of the computed energy decreasing versus iterations, illustrating that the algorithm is in practice stable and well-behaved. The same denoising test is performed with the model ${ }^{5}$ in Figure 4. We notice that, as expected, the proposed new method produces better results. Similar tests are shown with the noisy real image "Lena" in Figures 5 and 6 . Again, the proposed method gives better denoised result than with the ROF method, based on the values of the RMSE and of the SNR. Note that all the above mentioned denoising methods, including the proposed one, are different from the non-local means method ${ }^{33}$ which gives very good denoising results and also $f=u+v$ decompositions, but with a more expensive computational work).

We end this section with two image decomposition results into cartoon and texture applied to real images, obtained by the proposed method (Figures 7 and 8). The first image from Figure 7 is a fingerprint image, while the second image from 8 is part of the well-known Barbara image. As we can see, the proposed method gives very satisfactory results of separation into cartoon and texture.

In conclusion, we have illustrated that the proposed method gives better results than those obtained by the ROF model ${ }^{5}$ (although the ROF method ${ }^{5}$ is simpler). We think that the proposed results are comparable with those obtained by the other methods mentioned in Section 1, as approximations to the $(B V, G)$ model proposed by Y. Meyer. Theoretical results, including characterization of minimizers, will be analyzed in future work. 


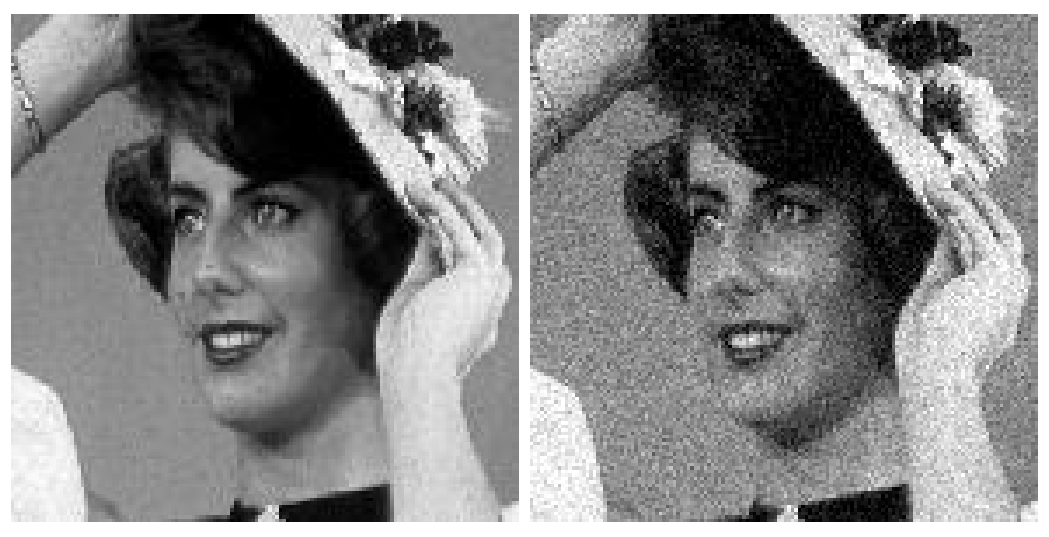

Figure 2. Real original image and its noisy version $f$.
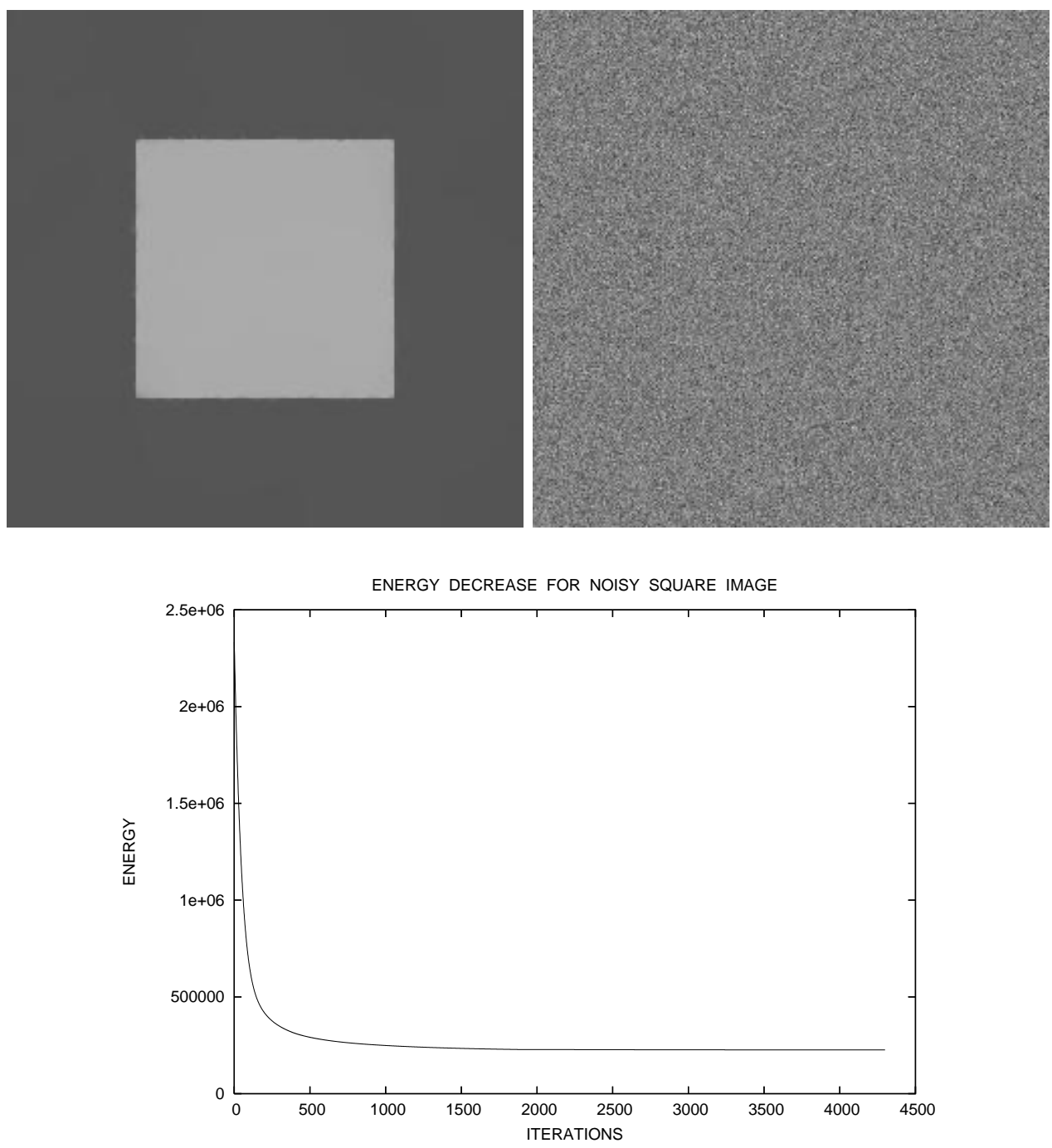

Figure 3. Top: denoised $u$ and residual $f-u$ by the proposed method. Bottom: energy decrease versus iterations. $\mathrm{RMSE}=0.0050, \mathrm{SNR}=29.0836$. 

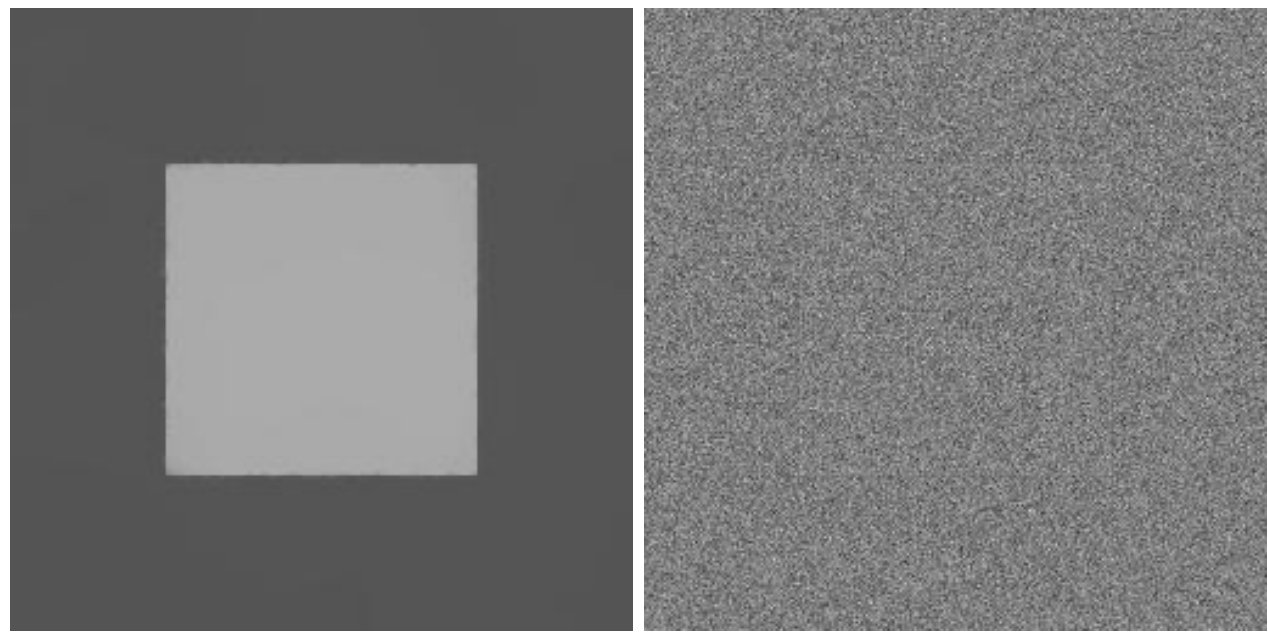

Figure 4. Denoised $u$ and residual $f-u$ by the ROF method for the synthetic noisy image. RMSE $=0.0053, \mathrm{SNR}=28.6534$.
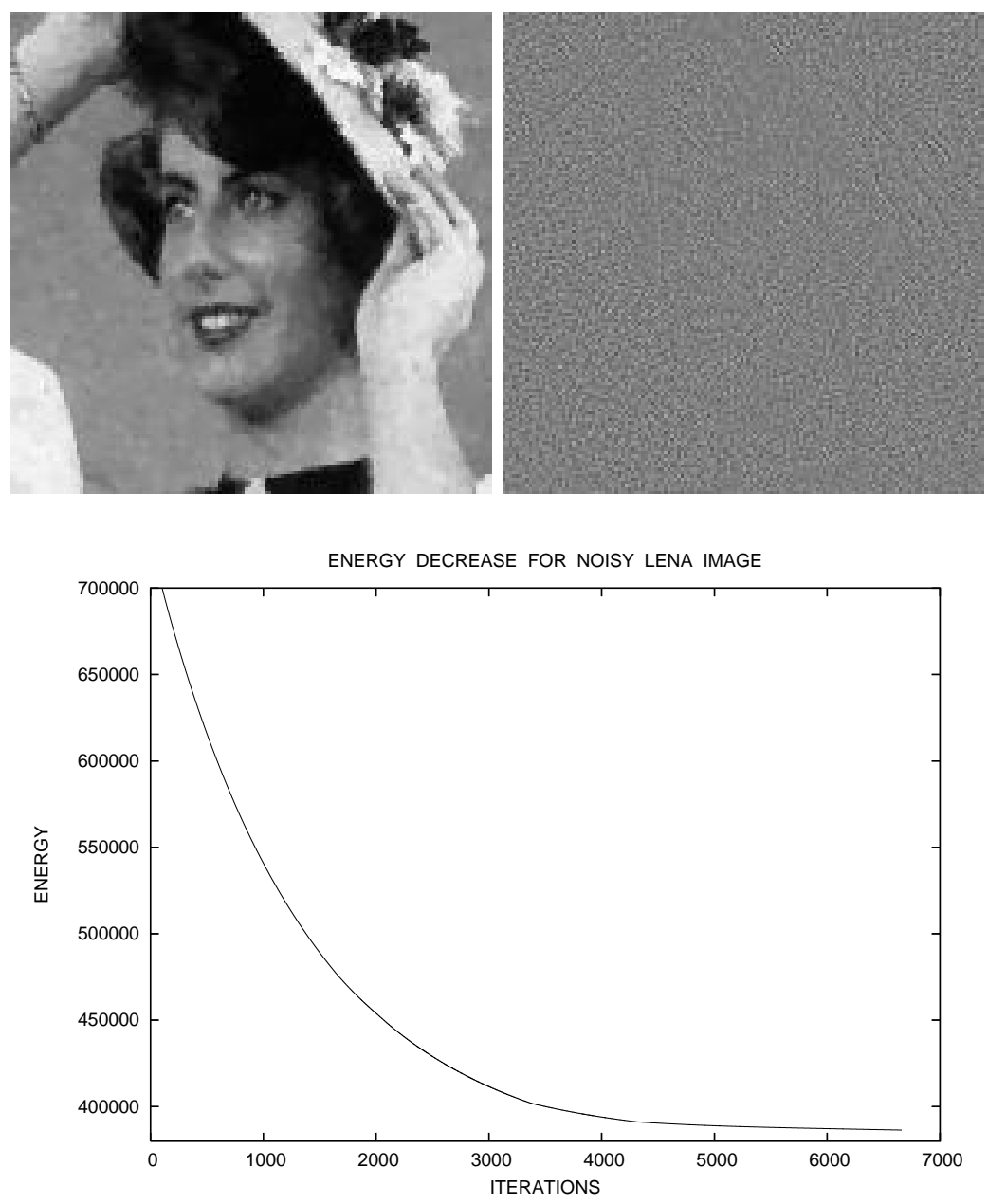

Figure 5. Top: denoised $u$ and residual $f-u$ by the proposed method for the real noisy Lena image. Bottom: energy decrease versus iterations. $\mathrm{RMSE}=0.0565, \mathrm{SNR}=17.9983$. 

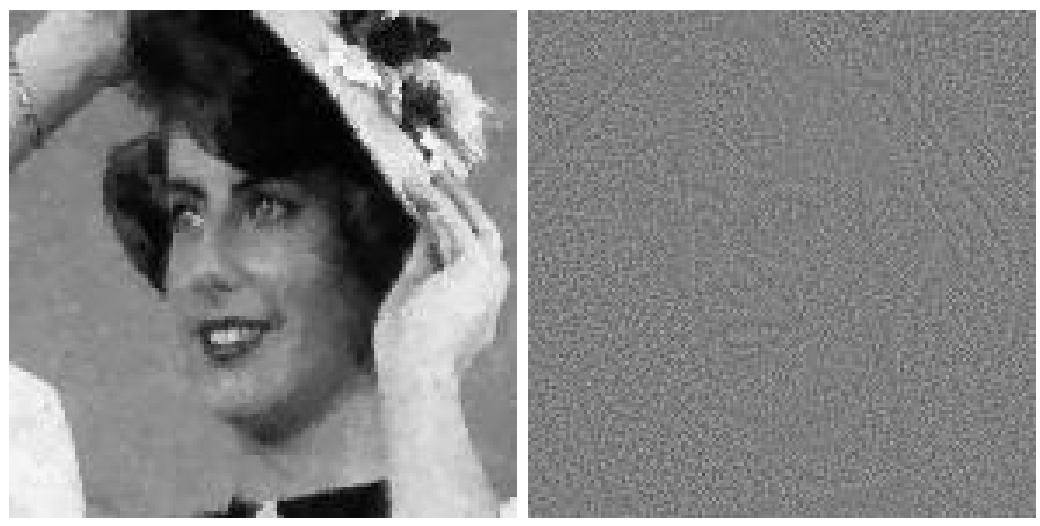

Figure 6. Denoised $u$ and residual $f-u$ by the ROF method for the real noisy Lena image. RMSE $=0.0567, \mathrm{SNR}=17.9744$.
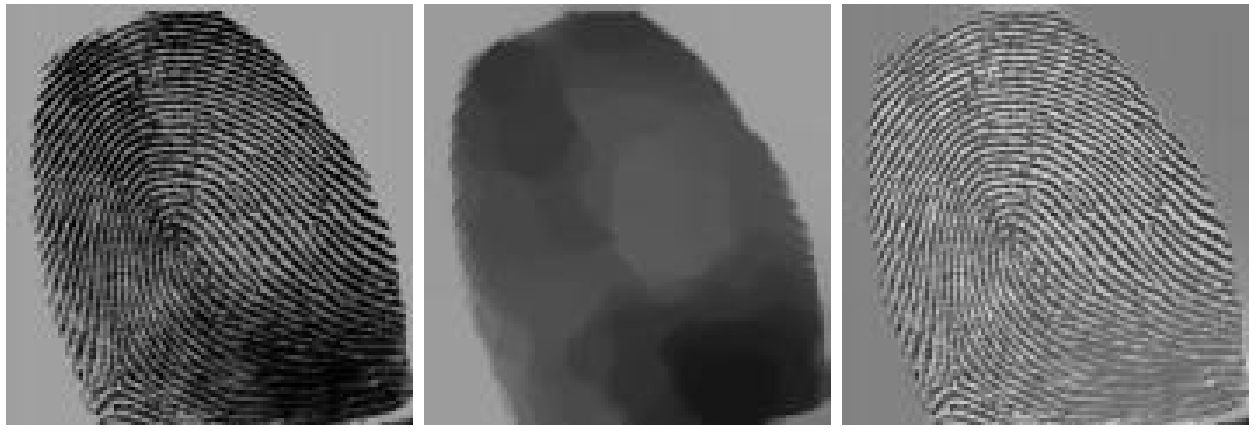

Figure 7. Left: initial textured image $f$. Middle: cartoon component $u$. Right: texture component $f-u \approx v$ (obtained by the proposed image decomposition model).
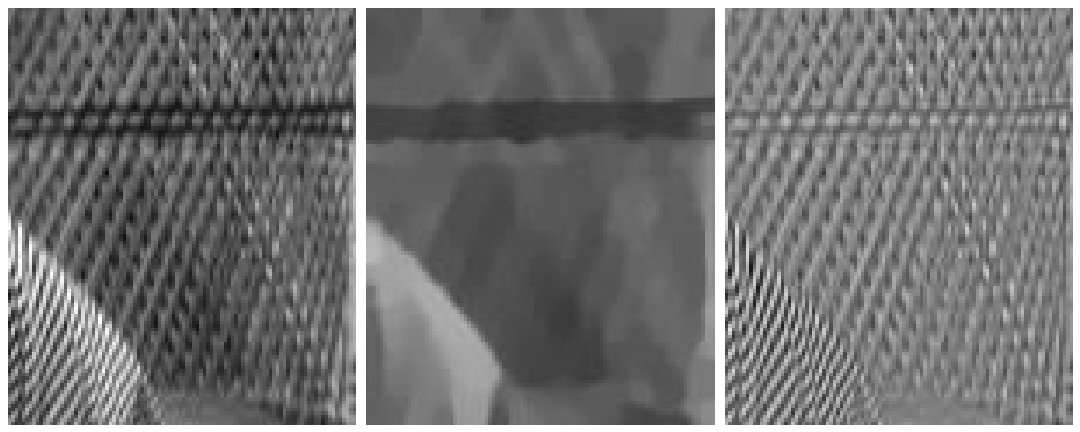

Figure 8. Left: initial textured image $f$. Middle: cartoon component $u$. Right: texture component $f-u \approx v$ (obtained by the proposed image decomposition model). 


\section{ACKNOWLEDGMENTS}

L.A.V. would like to thank the editors Charles A. Bouman, Eric L. Miller, and Ilya Pollak for their invitation to participate in the SPIE Electronic Imaging Conference "Computational Imaging V", San Jose, CA, January 2007.

This work has been supported in part by an Alfred P. Sloan fellowship (L.A.V.), by the National Institute of Health through the NIH Roadmap for Medical Research (Grant U54 RR021813 entitled Center for Computational Biology CCB), by the National Science Foundation Grants NSF ITR 0113439, NSF DMS 0312222.

Finally, both authors would like to thank Jean-Michel Morel for his useful remarks and suggestions made during several discussions and meetings at UCLA with the authors.

\section{REFERENCES}

1. Y. Meyer, Oscillating Patterns in Image Processing and Nonlinear Evolution Equations, Volume 22, AMS, 2001.

2. S.-C. Zhu and D. Mumford, "Prior learning and Gibbs reaction-diffusion," IEEE Transactions on PAMI 19(11), pp. 1236-1250, 1997.

3. S. Geman and D. Geman, "Stochastic relaxation, Gibbs distributions, and the Bayesian restoration of images," IEEE Transactions on PAMI 6(6), pp. 721-741, 1984.

4. D. Geman and G. Reynolds, "Constrained restoration and the recovery of discontinuities," IEEE T. on PAMI 14 (3), pp. 367-383, 1992.

5. L. I. Rudin, S. Osher, and E. Fatemi, "Nonlinear total variation based noise removal algorithms," Physica D: Nonlinear Phenomena 60(1-4), pp. 259-268, 1992.

6. A. Chambolle and P.-L. Lions, "Image recovery via total variation minimization and related problems," Numerische Mathematik 76(2), pp. 167-188, 1997.

7. L. Vese, "A study in the BV space of a denoising-deblurring variational problem," Applied Mathematics and Optimization 44(2), pp. 131-161, 2001.

8. G. Aubert and P. Kornprobst, Mathematical Problems in Image Processing: Partial Differential Equations and the Calculus of Variations, Springer, Applied Mathematical Sciences, Vol 147, 2006.

9. R. Acar and C. R. Vogel, "Analysis of bounded variation penalty methods for ill-posed problems," Inverse Problems 10(6), pp. 1217-1229, 1994.

10. L. C. Evans and R. F. Gariepy, Measure Theory and Fine Properties of Functions, CRC Press (Studies in Advance Mathematics), 1991.

11. F. Andreu-Vaillo, V. Caselles, and J. M. Mazon, Parabolic Quasilinear Equations Minimizing Linear Growth Functionals, Progress in Mathematics Vol. 223, Birkhauser, 2004.

12. L. Rudin, Images, Numerical Analysis of Singularities and Shock Filters, Ph.D. thesis, California Institute of Technology, Pasadena, CA, 1987.

13. L. I. Rudin and S. Osher, "Total variation based image restoration with free local constraints," in ICIP (1), pp. 31-35, 1994.

14. D. Strong and T. Chan, "Edge-preserving and scale-dependent properties of total variation regularization," Inverse Problems 19 (6), pp. S165-S187, 2003.

15. D. Mumford and B. Gidas, "Stochastic models for generic images," Quart. Appl. Math. 59, pp. 85-111, 2001.

16. Y. Gousseau and J.-M. Morel, "Are natural images of bounded variation ?," SIAM J. Num. Anal. 33(3), pp. 634-648, 2001.

17. L. Alvarez, Y. Gousseau, and J.-M. Morel, "Scales in natural images and a consequence on their bounded variation norm," LNCS 1682(3), pp. 247-258, 1999.

18. D. Mumford and J. Shah, "Boundary detection by minimizing functionals," in IEEE Computer Vision and Pattern Recognition (CVPR), pp. 22-26, 1985.

19. D. Mumford and J. Shah, "Optimal approximations by piecewise smooth functions and variational problems," Communications on Pure and Applied Mathematics XLII(5), pp. 577-685, 1988. 
20. L. A. Vese and S. J. Osher, "Modeling textures with total variation minimization and oscillating patterns in image processing," Journal of Scientific Computing 19(1-3), pp. 553-572, 2003.

21. L. A. Vese and S. J. Osher, "Image denoising and decomposition with total variation minimization and oscillatory functions," Journal of Mathematical Imaging and Vision 20(1-2), pp. 7-18, 2004.

22. L. C. Evans, Partial Differential Equations, AMS, GSM, Vol 19, 2002.

23. S. J. Osher, A. Solé, and L. A. Vese, "Image decomposition and restoration using total variation minimization and the $H^{-1}$ norm," Multiscale Modeling and Simulation 1(3), pp. 349-370, 2003.

24. J.-F. Aujol, G. Aubert, L. Blanc-Féraud, and A. Chambolle, "Image decomposition application to SAR images," in Scale-Space, pp. 297-312, 2003.

25. J.-F. Aujol, G. Aubert, L. Blanc-Féraud, and A. Chambolle, "Image decomposition into a bounded variation component and an oscillating component," Journal of Mathematical Imaging and Vision 22, pp. 71-88, Jan. 2005.

26. G. Aubert and J.-F. Aujol, "Modeling very oscillating signals. Application to image processing," Applied Mathematics and Optimization 51(2), pp. 163-182, 2005.

27. T. M. Le and L. A. Vese, "Image decomposition using total variation and $\operatorname{div}(\mathrm{BMO}), "$ Multiscale Modeling and Simulation 4(2), pp. 390-423, 2005.

28. W. Yin, D. Goldfarb, and S. Osher, "A comparison of total variation based texture extraction models," Journal of Visual Communication and Image Representation (to appear).

29. T. Le, L. Lieu, and L. Vese, "BV and dual of BV image decomposition models and minimization algorithms," UCLA CAM Report (05-13), 2005.

30. G. Aronsson, "Extension of functions satisfying Lipschitz conditions," Arkiv for Matematik 6(6), pp. 551$561,1967$.

31. R. Jensen, "Uniqueness of Lipschitz extensions - minimizing the sup norm of the gradient," Archive for Rational Mechanics and Analysis 123(1), pp. 51-74, 1993.

32. V. Caselles, J.-M. Morel, and C. Sbert, "An axiomatic approach to image interpolation," IEEE Transactions on Image Processing 7(3), pp. 376-386, 1998.

33. A. Buades, B. Coll, and J.-M. Morel, "A review of image denoising algorithms, with a new one," Multiscale Modeling and Simulation 4(2), pp. 490-530, 2005. 